\title{
Editorials
}

\section{Prosthesis-patient size: Measurement and clinical implications}

\author{
A. Marc Gillinov, MD \\ Eugene $\mathrm{H}$. Blackstone, $\mathrm{MD}^{\mathrm{a}, \mathrm{b}}$ \\ Leonardo L. Rodriguez, MD ${ }^{c}$
}

See related articles on pages 317 and 337.
From the Departments of Cardiothoracic Surgery, ${ }^{\mathrm{a}}$ Cardiovascular Medicine, ${ }^{\mathrm{c}}$ and Biostatistics, ${ }^{b}$ The Cleveland Clinic Foundation, Cleveland, Ohio.

Received for publication Sept 19, 2002; accepted for publication Oct 17, 2002.

Address for reprints: A. Marc Gillinov, MD, Department of Thoracic and Cardiovascular Surgery, The Cleveland Clinic Foundation/F25, 9500 Euclid Ave, Cleveland, OH 44195 (E-mail: gillinom@ccf. org).

J Thorac Cardiovasc Surg 2003;126:313-6

Copyright (C) 2003 by The American Association for Thoracic Surgery

0022-5223/2003\$30.00+0

doi:10.1016/S0022-5223(02)73223-6
"Mismatch can be considered to be present when the effective prosthetic valve area, after insertion into the patient, is less than that of a normal human valve. . occasionally it can be a severe problem because the patient may be hemodynamically and symptomatically worse after valve replacement."

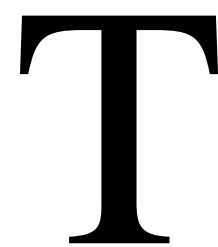

his is the definition of prosthesis-patient mismatch framed by Rahimtoola ${ }^{1}$ when he coined the term in 1978 . Rahimtoola suggested that the degree of prosthesis-patient mismatch could be quantified and that such quantification might aid in identifying patients at risk of clinical sequelae caused by this condition. DeCarlo and colleagues $^{2}$ seek to undertake these tasks in their report on small-sized Sorin Bicarbon prostheses (Sorin Biomedica, Saluggia, Italy). Although seemingly straightforward, application of Rahimtoola's definition requires examination and clarification of several complex concepts, including (1) measurement of prosthesis size, (2) measurement of patient size, (3) normalization of these values, (4) selection of referent values, and (5) assessment of the influence of prosthesis-patient size on clinical outcome.

\section{Prosthesis Size}

Prosthesis size can be based on either geometric dimensions or functional performance of the prosthesis (Table 1). Geometric expressions of prosthesis size include labeled size and internal orifice size. Functional expressions of prosthesis size include both in vitro and in vivo effective orifice area (EOA).

Although used frequently to assess the effect of prosthesis size on outcome, labeled valve size is limited in this regard. ${ }^{3}$ Manufacturer's labeled size refers inconsistently to diameter of the external sewing ring (mechanical prostheses), mounting ring (stented xenografts), or internal orifice (allografts and some stentless xenografts). Furthermore, valves of the same type (mechanical or bioprosthetic) and same labeled size from 2 different manufacturers nearly always have disparate geometric dimensions. ${ }^{3}$ Given these limitations, labeled valve size is a poor choice for analyzing the effect of prosthesis size on patient outcome. Recently, the International Organization for Standardization (ISO) addressed these concerns by recommending that manufacturers adopt a common standard for labeled prosthesis size. ${ }^{4}$ The ISO suggested that labeled prosthesis size should represent the tissue annulus diameter of the patient in whom the valve is to be implanted. However, it represents an external dimension of a prosthesis and not the internal orifice through which blood flows.

Unlike labeled prosthesis size or the ISO proposal, internal orifice size is a 


\section{TABLE 1. Measurements of prosthesis size}

Geometric
Manufacturer's labeled size
Internal orifice size
Diameter
Area
Functional
In vitro effective orifice area
Static
Dynamic
In vivo effective orifice area

dimension directly related to blood flow and is a fixed and reproducible geometric value obtained before valve insertion. Internal orifice size might be expressed as the internal diameter or the internal area, with the latter calculated by assuming a circular shape and disregarding hinge areas of mechanical valves and irregularities in bioprostheses; these features represent limitations in the use of internal orifice area to represent prosthesis size.

In vitro EOA is a functional determination of valve size. It varies considerably, depending on conditions and techniques of measurement and the type of prosthesis. In vitro EOA can be determined under static hydrodynamic conditions at a variety of flow rates or, alternatively, under dynamic conditions with variable pulsatile waveforms and flow rates. Artificial static EOA measurements performed under steady flow are poorly reproduced from laboratory to laboratory. Estimates of static EOA for bioprostheses vary by as much as $100 \%$ as the steady flow rate increases. Dynamic (pulsatile) in vitro EOA data are nonstandardized, unreproducible, and unavailable. For these reasons, in vitro EOA is generally unsuitable for assessment of the clinical effect of prosthesis size.

In vivo EOA has been used extensively to examine the relationship of prosthesis size, patient size, and clinical outcome. ${ }^{5}$ Obtained by means of echocardiography after implantation, in vivo EOA can be measured serially. In vivo EOA is calculated from the continuity equation:

Aortic valve area $=\left(\mathrm{A}_{\text {lvot }} \times \mathrm{VTI}_{\text {lvot }}\right) / \mathrm{VTI}_{\text {transvalvular }}$

where $A_{\text {lvot }}$ is the area of the left ventricular outflow tract (LVOT) just before the valve, $\mathrm{VTI}_{\text {lvot }}$ is the velocity-time integral of the pulsed Doppler velocity at the outflow tract level, and $\mathrm{VTI}_{\text {transvalvular }}$ is calculated from the continuous wave Doppler velocity through the prosthetic valve.

The area obtained with this equation is the EOA, which is smaller than the anatomic area and corresponds to the smallest area of the jet as it exits the valve (vena contracta). The shape of the inlet and the size of the orifice affect the ratio between the anatomic orifice area and the EOA (coefficient of orifice contraction). ${ }^{6}$ The continuity equation assumes that flow coming into the narrowed orifice has a flat profile; the actual flow profile varies between prostheses, with mechanical prostheses having the greatest variance from a flat profile.

A functional determination of prosthesis size, in vivo EOA might vary widely, depending on a number of factors related to measurement technique and patient state. Variability can be introduced in the measurement of the LVOT diameter or the velocity at the outflow tract. ${ }^{7}$ In vivo EOA values for bileaflet valves might be underestimated when evaluated by means of echocardiography because of localized high-velocity jets. Poor echocardiographic windows and suboptimal Doppler recordings with improper alignment of the Doppler scan with the direction of flow might also affect the accuracy of the method.

Although this method is, in theory, independent of flow, flow dependency does exist. ${ }^{8}$ Most important, in vivo EOA varies considerably with the state of the patient. ${ }^{8}{ }^{8}$ In vivo EOA might change from moment to moment with patient activity, cardiac output and blood pressure, and dynamics of the LVOT, as well as intrinsic prosthesis properties. Estimates of bioprosthesis in vivo EOA are particularly sensitive to the level of patient activity (rest vs exercise), with measured in vivo EOA tending to increase with exercise. ${ }^{10}$ In vivo EOA values have been observed to change during the first year after implantation as hemodynamic data change. ${ }^{11,12}$

\section{Patient Size}

Assessment of the effect of aortic prosthesis size on outcome should take into account the size of the patient. Patient size has been quantified on the basis of height, weight, and body surface area (BSA). In the contemporary literature most studies use BSA, and there is growing agreement that this is the most suitable measurement in this regard.

\section{Normalization}

Normalization of any expression of prosthesis size can be made to body size. This concept is used to account for both prosthesis size and patient size and, furthermore, to compare values in individual patients with those in the larger population. When BSA is used to express patient size, 2 common normalization methods are used to express the relationship between prosthesis and patient size: indexing and standardization.

An indexed valve dimension is obtained by dividing that dimension by the BSA. Any valve dimension, whether geometric or functional, can be indexed. For example, both the geometric internal prosthesis area and the in vivo EOA might be expressed as indexed values (ie, square centimeter per square meter of BSA).

The concept of standardization enables comparison of individual patient values with those obtained from a population. Use of dimensionless standardized values is based on 


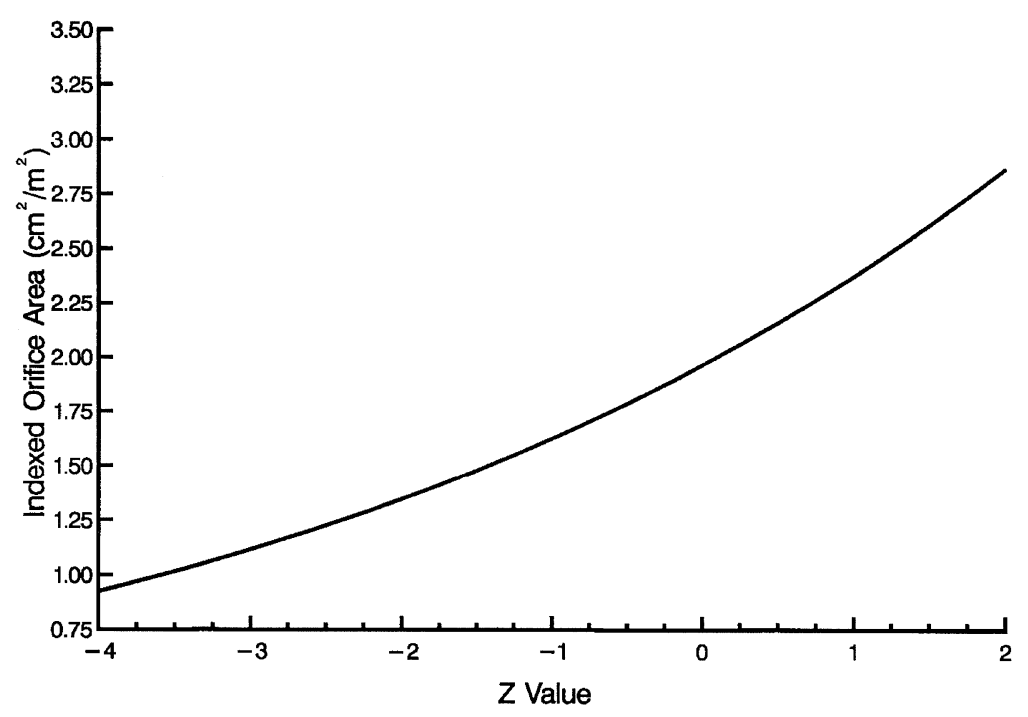

Figure 1. Indexed orifice area versus $Z$ value for the aortic valve. The relationship is approximately logarithmic.

the fact that native annulus size exhibits considerable variability among normal individuals of any given size. Rimoldi and $\mathrm{Lev}^{13}$ introduced the idea that normality and abnormality could be expressed in quantitative terms as the number of SDs away from normal size a given patient's cardiac structures measure. This is the concept of the $\mathrm{Z}$ value.

In the current context $\mathrm{Z}$ is the number of SDs between the patient's prosthetic aortic valve size and the mean normal native aortic valve size for a patient of a given BSA. Capps and colleagues ${ }^{14}$ have provided the reference values for the distribution of native aortic valve diameters in the general population. By means of these data and measured prosthetic internal orifice diameters, the standardized orifice size, or $\mathrm{Z}$ value, might be determined for any patient receiving a prosthetic aortic valve.

These concepts of normalization enable expression of prosthesis-patient size. Prosthesis-patient size is a means of quantifying the relationship between prosthesis size and patient size. As stated above, any term representing prosthesis size (in vivo EOA or internal orifice area) can be indexed to patient BSA. Furthermore, prosthesis-patient size might be related to mean values in the general population by means of standardization (the $\mathrm{Z}$ value). There is an approximately logarithmic relationship between indexed and standardized prosthesis-patient size (Figure 1).

\section{Referent Values}

There is interest in providing referent values for prosthesispatient size to aid surgeons in prosthesis selection, such that each patient receives a prosthesis of sufficient size. De Carlo and colleagues ${ }^{2}$ seek to accomplish this task for the Sorin Bicarbon prosthesis. In theory referent values could be generated from both geometric and functional measures of prosthesis size. Although they do not address directly the function of a prosthesis in a given patient, referent values on the basis of geometric measures of prosthesis size are determined before implantation, have little variability, and are independent of hemodynamic state. Pibarot and colleagues ${ }^{15}$ have suggested that rather than using a geometric prosthesis dimension as a fixed reference for prosthesis size, a fixed referent value of in vivo EOA should be used. They termed this "projected EOA." Such referent values were obtained from informal meta-analysis of literature sources. This strategy has several important drawbacks: it suffers from flow dependency, a large scatter in the data, rest versus exercise differences, and limited availability of data for each prosthesis size and model. Thus referent values on the basis of geometric determinants of prosthesis size are preferable at this time.

\section{Prosthesis-Patient Size and Clinical Outcome}

Given the broad definition of prosthesis-patient mismatch and the methodology for determination of prosthesis-patient size, what is the clinical importance of these concepts? Among cardiac surgeons and cardiologists, there is considerable interest in aortic prosthesis size, with the common notion being that placement of a relatively small valve might jeopardize outcome. The reasoning behind the notion that bigger valves are better seems logical. ${ }^{1,5} \mathrm{~A}$ larger prosthetic aortic valve will have superior hemodynamic performance, which is manifested by lower transvalvular gradients. This should result in decreased left ventricular work and more rapid and complete regression of left ventricular hypertrophy. These factors, in turn, are purported to improve clinical status and prolong survival. Although sup- 
porting data are limited, these statements have been accepted as fact.

Until recently, the effect of prosthesis-patient size on survival was controversial. Although some studies suggested that smaller prosthesis-patient size jeopardized early or late survival, ${ }^{16}$ others did not support this finding. ${ }^{17,18}$ Recently, 3 large studies, each containing more than 1000 patients, have helped to clarify the effect of prosthesispatient size on survival. In a study of 1129 patients, Hanayama and colleagues ${ }^{19}$ found that prosthesis-patient size did not influence survival, New York Heart functional class, or left ventricular mass index. Rao and associates ${ }^{20}$ examined a cohort of 2981 patients undergoing aortic valve replacement; they observed that smaller prosthesis-patient size was associated with greater operative mortality but did not affect overall 12-year survival.

The question of prosthesis-patient size and mortality after aortic valve replacement was addressed by Blackstone and coworkers. ${ }^{21}$ In this study, including data on 13,258 aortic valve replacements from 9 data sources, 30-day mortality increased $1 \%$ to $2 \%$ when indexed orifice area decreased to less than $1.2 \mathrm{~cm}^{2} / \mathrm{m}^{2}$ or standardized orifice size decreased to less than $-2.5 \mathrm{Z}$. However, no expression of prosthesis-patient size was associated with reduced intermediate-term (0.5-5 years) or late-term (5-15 years) survival. In contrast, patient risk factors, such as age, had a profound effect on survival.

Although small prosthesis-patient size has a small effect on operative mortality and no important effect on late survival, this does not rule out a possible influence on other indices of clinical outcome. It is possible that highly active and larger individuals might benefit from superior hemodynamics that might be associated with greater prosthesispatient size. This assertion is very difficult to prove. Large studies addressing this issue are warranted.

Aortic valve replacement improves survival in patients with severe aortic stenosis. Assessment of the effect of prosthesis-patient size in such patients requires adoption of standard methodology. Indexed orifice area and standardized orifice size depend on geometric measurements obtained before implantation, are reproducible, and allow comparison with the normal population. This is a preferred method for assessing prosthesis-patient size. Indexed in vivo $\mathrm{EOA}$, a functional measurement obtained in an individual patient in a given hemodynamic state, provides complementary information. Future studies of the effect of prosthesis-patient size should, where possible, include both types of measurement. Such a strategy will enable more complete characterization of the effect of prosthesis-patient size on clinical outcome.

\section{References}

1. Rahimtoola SH. The problem of valve prosthesis-patient mismatch. Circulation. 1978;58:20-4

2. De Carlo M, Milano AD, Nardi C, Mecozzi G, Bortolotti U. Serial Doppler echocardiographic evaluation of small-sized Sorin Bicarbon prostheses. J Thorac Cardiovasc Surg. 2003;126:337-43.

3. Christakis GT, Buth KJ, Goldman BS, et al. Inaccurate and misleading valve sizing: a proposed standard for valve size nomenclature. Ann Thorac Surg. 1998;66:1198-203.

4. International Organization for Standardization. Cardiovascular implants - cardiac valve prostheses. International Organization for Standardization; 2002. Committee draft ISO/CD5840.

5. Pibarot P, Dumesnil JG. Hemodynamic and clinical impact of prosthesis-patient mismatch in the aortic valve position and its prevention. J Am Coll Cardiol. 2000;36:1131-41.

6. Flaschskampf FA, Weyman AE, Guerrero JL, Thomas JD. Influence of orifice geometry and flow rate on effective valve area: an in vitro study. J Am Coll Cardiol. 1991;15:1173-80.

7. Badano L, Mocchegiani R, Bertoli D, et al. Normal echocardiographic characteristics of the Sorin Bicarbon bileaflet prosthetic heart valve in the mitral and aortic positions. J Am Soc Echocardiogr. 1997;10:63243.

8. Dumesnil JG, Honos GN, Lemieux M, Beauchemin J. Validation and applications of indexed aortic prosthetic valve areas calculated by Doppler echocardiography. J Am Coll Cardiol. 1990;16:637-43.

9. Barratt-Boyes BG. The timing of operation in valvular insufficiency. J Card Surg. 1987;2:435-52.

10. Pibarot P, Dumesnil JG, Jobin J, Cartier P, Honos G, Durand LG. Hemodynamic and physical performance during maximal exercise in patients with an aortic bioprosthetic valve: comparison of stentless versus stented bioprostheses. J Am Coll Cardiol. 1999;34:1609-17.

11. Dumesnil JG, LeBlanc MH, Cartier P, et al. Hemodynamic features of the freestyle aortic bioprosthesis compared with stented bioprosthesis. Ann Thorac Surg. 1998;66(suppl S):S130-3.

12. Cartier PC, Dumesnil JG, Metras J, et al. Clinical and hemodynamic performance of the freestyle aortic root bioprosthesis. Ann Thorac Surg. 1999;67:345-51.

13. Rimoldi H, Lev M. A note on the concept of normality and abnormality in quantitation of pathologic findings in congenital heart disease. Pediatr Clin North Am. 1963;10:589-91.

14. Capps SB, Elkins RC, Fronk DM. Body surface area as a predictor of aortic and pulmonary valve diameter. $J$ Thorac Cardiovasc Surg. 2000;119:975-82.

15. Pibarot P, Dumesnil JG, Cartier PC, Metras J, Lemieux MD. Patientprosthesis mismatch can be predicted at the time of operation. Ann Thorac Surg. 2001;71(suppl S):S265-8.

16. Kratz JM Sade RM, Crawford FA, Crumbley AJ, Stroud MR. The risk of small St. Jude aortic valve prostheses. Ann Thorac Surg. 1994;57: 1114-9.

17. Sawant D, Singh AK, Feng WC Bert AA, Rotenberg F. St Jude Medical cardiac valves in small aortic roots: follow-up to sixteen years. J Thorac Cardiovasc Surg. 1997;113:499-509.

18. Medalion B, Blackstone EH, Lytle BW, White J, Arnold JH, Cosgrove DM. Aortic valve replacement: is valve size important? J Thorac Cardiovasc Surg. 2000;119:963-74.

19. Hanayama N, Christakis GT, Mallidi HR, et al. Patient prosthesis mismatch is rare after aortic valve replacement: valve size may be irrelevant. Ann Thorac Surg. 2002;73:1822-9.

20. Rao V, Jamieson WRE, Ivanov J, Armstrong S, David TE. Prosthesispatient mismatch affects survival after aortic valve replacement. Circulation. 2000;102(suppl III):III-5-9.

21. Blackstone EH, Cosgrove DM, Jamieson WRE, et al. Prosthesis size and long-term survival after aortic valve replacement. Presented at the 80th Annual Meeting of the American Association for Thoracic Surgery; Toronto, Ontario, Canada, 2000. 